\title{
Forecasting biodiversity in breeding birds using best practices
}

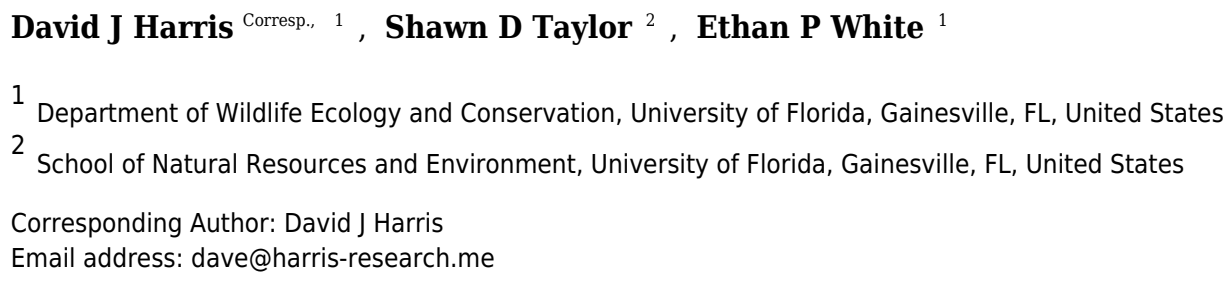

Biodiversity forecasts are important for conservation, management, and evaluating how well current models characterize natural systems. While the number of forecasts for biodiversity is increasing, there is little information available on how well these forecasts work. Most biodiversity forecasts are not evaluated to determine how well they predict future diversity, fail to account for uncertainty, and do not use time-series data that captures the actual dynamics being studied. We addressed these limitations by using best practices to explore our ability to forecast the species richness of breeding birds in North America. We used hindcasting to evaluate six different modeling approaches for predicting richness. Hindcasts for each method were evaluated annually for a decade at 1,237 sites distributed throughout the continental United States. All models explained more than $50 \%$ of the variance in richness, but none of them consistently outperformed a baseline model that predicted constant richness at each site. The best practices implemented in this study directly influenced the forecasts and evaluations. Stacked species distribution models and "naive" forecasts produced poor estimates of uncertainty and accounting for this resulted in these models dropping in the relative performance compared to other models. Accounting for observer effects improved model performance overall, but also changed the rank ordering of models because it did not improve the accuracy of the "naive" model. Considering the forecast horizon revealed that the prediction accuracy decreased across all models as the time horizon of the forecast increased. To facilitate the rapid improvement of biodiversity forecasts, we emphasize the value of specific best practices in making forecasts and evaluating forecasting methods. 
- Forecasting biodiversity in breeding birds

2

3

4

5

${ }^{1}$ Department of Wildlife Ecology and Conservation, University of Florida, Gainesville,

7 FL, United States

$8^{2}$ School of Natural Resources and Environment, University of Florida Gainesville, FL, 9 United States

\section{using best practices}

David J. Harris ${ }^{1}$ (corresponding author)

Shawn D. Taylor ${ }^{2}$

Ethan P. White ${ }^{1}$ 


\section{Abstract}

Biodiversity forecasts are important for conservation, management, and evaluating how well current models characterize natural systems. While the number of forecasts for biodiversity is increasing, there is little information available on how well these forecasts work. Most biodiversity forecasts are not evaluated to determine how well they predict future diversity, fail to account for uncertainty, and do not use time-series data that captures the actual dynamics being studied. We addressed these limitations by using best practices to explore our ability to forecast the species richness of breeding birds in North America. We used hindcasting to evaluate six different modeling approaches for predicting richness. Hindcasts for each method were evaluated annually for a decade at 1,237 sites distributed throughout the continental United States. All models explained more than $50 \%$ of the variance in richness, but none of them consistently outperformed a baseline model that predicted constant richness at each site. The best practices implemented in this study directly influenced the forecasts and evaluations. Stacked species distribution models and "naive" forecasts produced poor estimates of uncertainty and accounting for this resulted in these models dropping in the relative performance compared to other models. Accounting for observer effects improved model performance overall, but also changed the rank ordering of models because it did not improve the accuracy of the "naive" model. Considering the forecast horizon revealed that the prediction accuracy decreased across all models as the time horizon of the forecast increased. To facilitate the rapid improvement of biodiversity forecasts, we emphasize the value of specific best practices in making forecasts and evaluating forecasting methods.

\section{Introduction}

Forecasting the future state of ecological systems is increasingly important for planning and management, and also for quantitatively evaluating how well ecological models 
capture the key processes governing natural systems (Clark et al. 2001, Dietze 2017, Houlahan et al. 2017). Forecasts regarding biodiversity are especially important, due to biodiversity's central role in conservation planning and its sensitivity to anthropogenic effects (Cardinale et al. 2012, Díaz et al. 2015, Tilman et al. 2017). High-profile studies forecasting large biodiversity declines over the coming decades have played a large role in shaping ecologists' priorities (as well as these of policymakers; e.g. IPCC 2014), but it is inherently difficult to evaluate such long-term predictions before the projected biodiversity declines have eccurred.

Previous efforts to predict future patterns of terrestrial species richness, and diversity more generally, have focused primarily on building species distributions models (SDMs; Thomas et al. 2004, Thuiller et al. 2011, Urban 2015). In general, these models describe individual species' occurrence patterns as functions of the environment. Given forecasts for environmental conditions, these models can predict where each species will occur in the future. These species-level predictions are then combined ("stacked") to generate forecasts for species richness (e.g. Calabrese et al. 2014). Alternatively, models that directly relate spatial patterns of species richness to environment conditions have been developed and generally perform equivalently to stacked SDMs (Algar et al. 2009, Distler et al. 2015). This approach is sometimes referred to as "macroecological" modeling, because it models the larger-scale pattern (richness) directly (Distler et al. 2015).

Despite the emerging interest in forecasting species richness and other aspects of biodiversity (Jetz et al. 2007, Thuiller et al. 2011), little is known about how effectively we can anticipate these dynamics. This is due in part to the long time scales over which many ecological forecasts are applied (and the resulting difficulty in assessing whether the predicted changes occurred; Dietze et al. 2016). What we do know comes from a small number of hindcasting studies, where models are built from different time periods and evaluated on their ability to predict biodiversity patterns in contemporary (Algar et 
al. 2009, Distler et al. 2015) or historic (Blois et al. 2013, Maguire et al. 2016) periods not used for model fitting. These studies are a valuable first step, but lack several components that are important for developing forecasting models with high predictive accuracy, and for understanding how well different methods can predict the future. These "best practices" for effective forecasting and evaluation (Box 1) broadly involve: 1) expanding the use of data to include biological and environmental time-series (Tredennick et al. 2016); 2) accounting for uncertainty in observations and processes, (Yu et al. 2010, Harris 2015); and 3) conducting meaningful evaluations of the forecasts by hindcasting, archiving short-term forecasts, and comparing forecasts to baselines to determine whether the forecasts are more accurate than assuming the system is basically static (Perretti et al. 2013).

In this paper, we attempt to forecast the species richness of breeding birds at over 1,200 of sites located throughout North America, while following best practices for ecological forecasting (Box 1). To do this, we combine 32 years of time-series data on bird distributions from annual surveys with monthly time-series of climate data and satellite-based remote-sensing. Datasets that span a time scale of 30 years or more have only recently become available for large-scale time-series based forecasting. A dataset of this size allows us to model and assess changes a decade or more into the future in the presence of shifts in environmental conditions on par with predicted climate change. We compare traditional distribution modeling based approaches to spatial models of species richness, time-series methods, and two simple baselines that predict constant richness for each site, on average (Figure 1). All of our forecasting models account for uncertainty and observation error, are evaluated across different time lags using hindcasting, and are publicly archived to allow future assessment. We discuss the implications of these practices for our understanding of, and confidence in, the resulting forecasts, and how we can continue to build on these approaches to improve ecological forecasting in the future. 


\section{A. Single-site models}
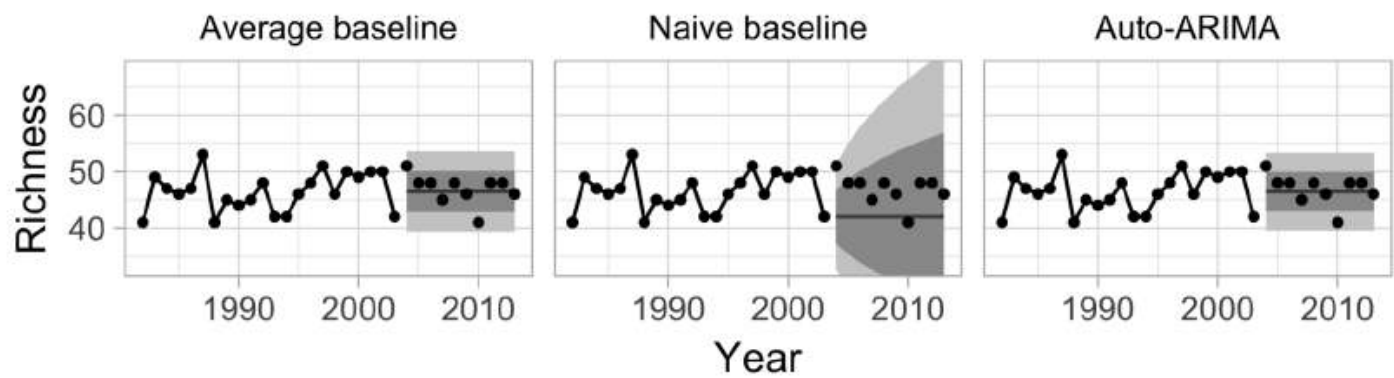

\section{B. Environmental models}
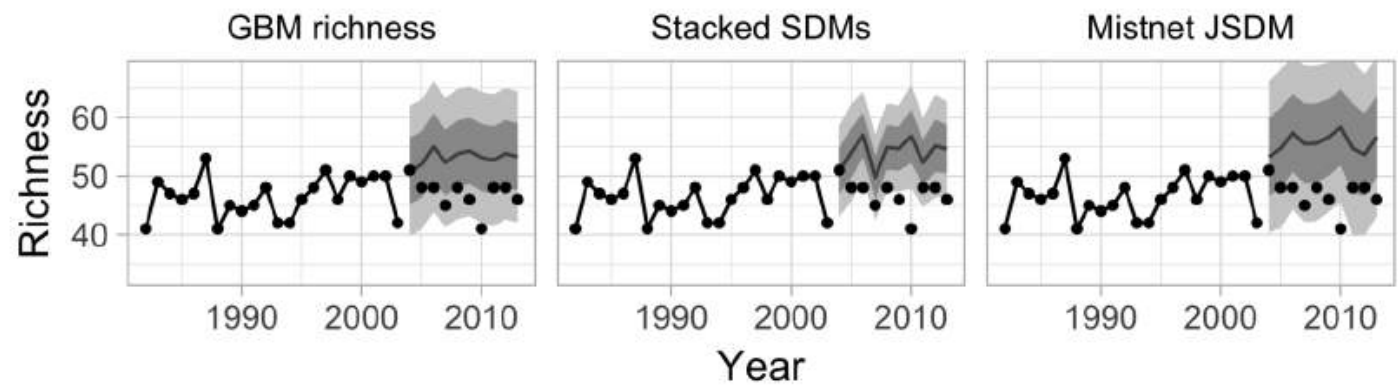

Figure 1: Example predictions from six forecasting models for a single site. Data from 1982 through 2003, connected by solid lines, were used for training the models; the remaining points were used for evaluating the models' forecasts. In each panel, point estimates for each year are shown with lines; the darker ribbon indicates the $68 \%$ prediction interval (1 standard deviation of uncertainty), and the lighter ribbon indicates the $95 \%$ prediction interval. A. Single-site models were trained independently on each site's observed richness values. The first two models ("average" and "naive") served as baselines. B. The environmental models were trained to predict richness based on elevation, climate, and NDVI; the environmental models' predictions change from year to year as environmental conditions change. 


\section{Methods}

We evaluated 6 types of forecasting models (Table 1) by dividing the 32 years of data into 22 years of training data and 10 years of data for evaluating forecasts using hindcasting. Here we use definitions from meteorology, where a hindcast is generally any prediction for an event that has already happened, while forecasts are predictions for actual future events (Jolliffe and Stephenson 2003). We also made long term forecasts by using the full data set for training and making forecasts through the year 2050. For both time frames, we made forecasts using each model with and without correcting for observer effects, as described below.

\section{Data}

Richness data. Bird species richness was obtained from the North American Breeding Bird Survey (BBS) (Pardieck et al. 2017) using the Data Retriever Python package (Morris and White 2013, Senyondo et al. 2017) and rdataretriever R package (McGlinn et al. 2017). BBS observations are three-minute point counts made at 50 fixed locations along a $40 \mathrm{~km}$ route. Here we denote each route as a site and summarize richness as the total species observed at all 50 locations in each surveyed year. Prior to summarizing the data was filtered to exclude all nocturnal, cepuscular, $\equiv$ aquatic species (since these species are not well sampled by BBS methods; Hurlbert and White 2005), as well as unidentified species, and hybrids. All data from surveys that did not meet BBS quality criteria were also excluded.

We used observed richness values from 1982 (the first year of complete environmental data) to 2003 to train the models, and from 2004 to 2013 to test their performance. We only used BBS routes from the continental United States (i.e. routes where climate data was available PRISM Climate Group (2004)), and we restricted the analysis to routes that were sampled during $70 \%$ of the years in the training period (i.e., routes with at least 16 annual observations). The resulting dataset included 34,494 annual surveys of 
1,279 unique sites, and included 385 species. Site-level richness varied from 8 to 91 with an average richness of 51 species.

Past environmental data. Environmental data included a combination of elevation, bioclimatic variables and a remotely sensed vegetation index (the normalized difference vegetation index; NDVI), all of which are known to influence richness and distribution in the BBS data (Kent et al. 2014). For each year in the dataset, we used the $4 \mathrm{~km}$ resolution PRISM data (PRISM Climate Group 2004) to calculate eight bioclimatic variables identified as relevant to bird distributions (Harris 2015): mean diurnal range, isothermality, max temperature of the warmest month, mean temperature of the wettest quarter, mean temperature of the driest quarter, precipitation seasonality, precipitation of the wettest quarter, and precipitation of the warmest quarter. These variables were calculated for the 12 months leading up to the annual survey (July-June) as opposed to the calendar year. Satellite-derived NDVI, a primary correlate of richness in BBS data (Hurlbert and Haskell 2002), was obtained from the NDIV3g dataset with an $8 \mathrm{~km}$ resolution (Pinzon and Tucker 2014) and was available from 1981-2013. Average summer (April, May, June) and winter (December, January, Feburary) NDVI values were used as predictors. Elevation was from the SRTM 90m elevation dataset (Jarvis et al. 2008) obtained using the R package raster (Hijmans 2016). Because BBS routes are 40-km transects rather than point counts, we used the average value of each environmental variable within a $40 \mathrm{~km}$ radius of each BBS route's starting point.

Future environmental projections. In addition to the analyses presented here, we have also generated and archived long term forecasts from 2014-2050. This will allow future researchers to assess the performance of our six models on longer time horizons as more years of BBS data become available. Precipitation and temperature were forecast using the CMIP5 multi-model ensemble dataset (Brekke et al. 2013). downscaled model runs (Brekke et al. 2013, see Table S1) using the RCP6.0 scenario were averaged together to create a single ensemble used to calculate the bioclimatic 
variables for North America. For NDVI, we used the per-site average values from 2000-2013 as a simple forecast. For observer effects (see below), each site was set to have zero observer bias. The predictions have been archived at (Harris et al. 2017b).

\section{Accounting for observer effects}

Observer effects are inherent in large data sets collected by different observers, and are known to occur in BBS (Sauer et al. 1994). For each forecasting approach, we trained two versions of the corresponding model: one with corrections for differences among observers, and one without (Figure 2). We estimated the observer effects (and associated uncertainty about those effects) using a linear mixed model, with observer as a random effect, built in the Stan probabilistic programming language (Carpenter et al. 2017). Because observer and site are strongly related (observers tend to repeatedly sample the same site), site-level random effects were included to ensure that inferred deviations were actually observer-related (as opposed to being related to the sites that a given observer happened to see). The resulting model is described mathematically and with code in Supplement S1. The model partitions the variance in observed richness values into site-level variance, observer-level variance, and residual variance (e.g. variation within a site from year to year).

Across our six modeling approaches (described below), we used estimates from the observer model in three different ways. First, the expected values for site-level richness were used directly as our "average" baseline model (see below). For the two models that made species-level predictions, the estimated observer effects were included alongside the environmental variables as predictors. Finally, we trained the remaining models to predict observer-corrected richness values (i.e. observed richness minus the observer effect, or the number of species that would have been recorded by a "typical" observer). Since the site-level and observer-level random effects are not known precisely, we represented the range of possible values using 500 Monte Carlo samples from the 
A. Without observer model
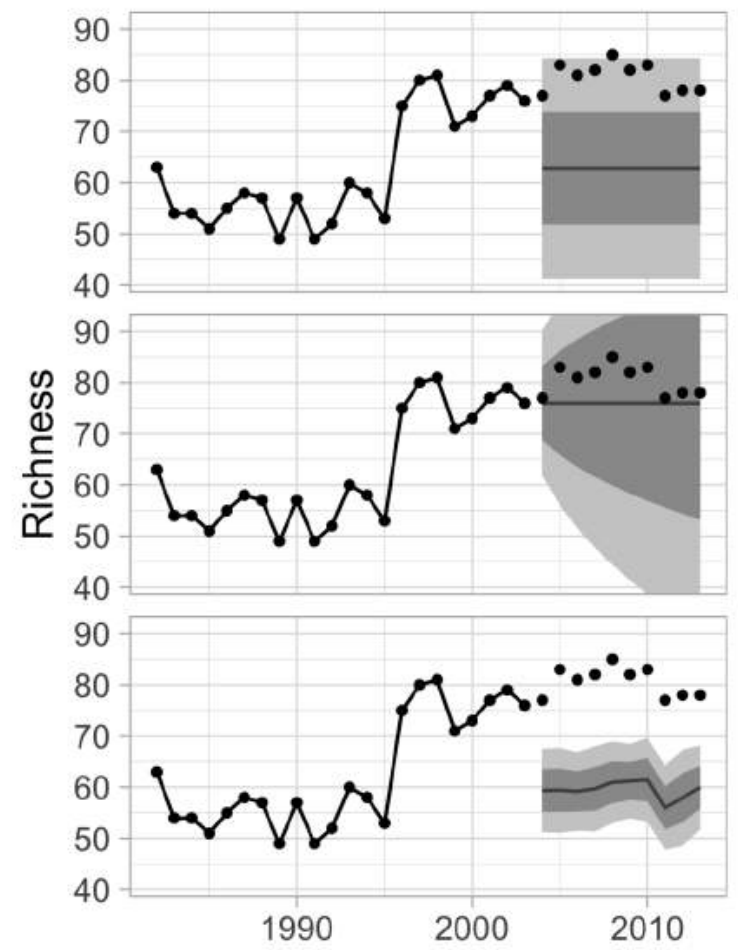

B. With observer model
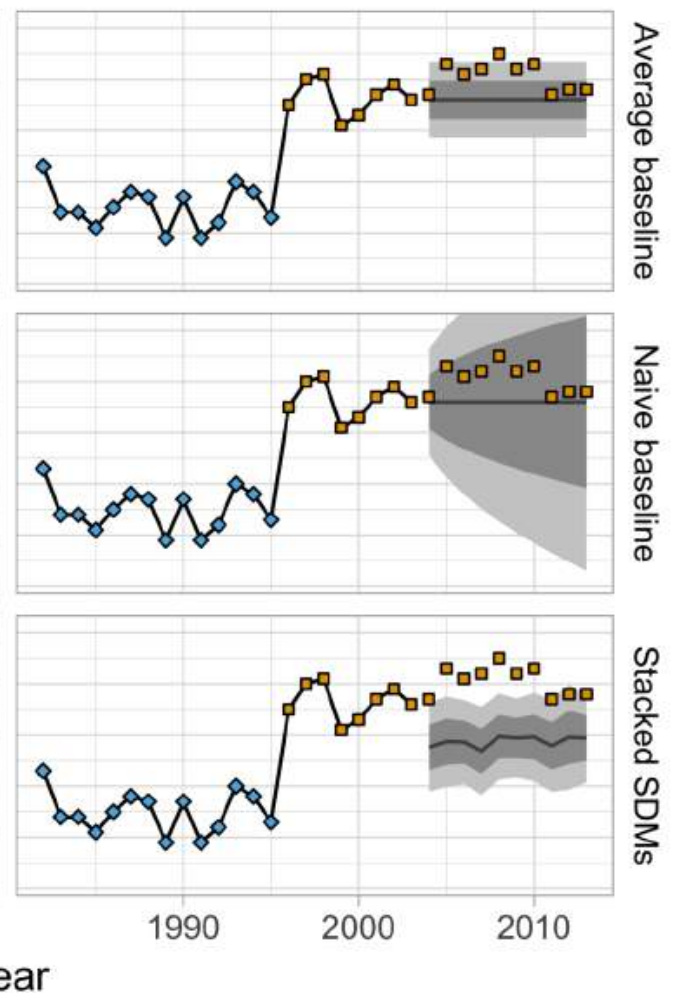

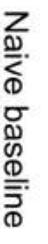

Figure 2: A. Model predictions for Pennsylvania route 35 when all observers are treated the same (black points). B. Model predictions for the same route when accounting for systematic differences between observers (represented by the points' colors). In this example most models are made more robust to observer turnover by including an observer model. Note that the "naive" model is less sensitive to observer turnover, and does not benefit as much from modeling it.

\section{Models: site-level models}

Three of the models used in this study were fit to each site separately, with no environmental information (Table 1). These models were fit to each BBS route twice: once using the residuals from the observer model, and once using the raw richness values. When correcting for observer effects, we averaged across 500 models that were fit separately to the 500 Monte Carlo estimates of the observer effects, to account for 
Table 1: Six forecasting models. Single-site models were trained site-by-site, without environmental data. Environmental models were trained at the continental scale, using only environmental variables (as opposed to site or time series information) as predictors. Most of the models were trained to predict richness directly. This mirrors the standard application of these techniques. Separate random forest SDMs were fit for each species and used to predict the probability of that species occurring at each site. The specieslevel probabilities at a site were summed to predict richness. The mistnet JSDM was trained to predict the full species composition at each site, and the number of species in its predictions was used as an estimate of richness.

\begin{tabular}{|c|c|c|c|c|}
\hline \multirow[b]{2}{*}{ Model } & \multirow[b]{2}{*}{ Response variable } & \multicolumn{3}{|c|}{ Predictors } \\
\hline & & Site id & Time & Environment \\
\hline \multicolumn{5}{|l|}{ Single-site models } \\
\hline Average baseline & richness & $\checkmark$ & & \\
\hline Naive baseline & richness & $\checkmark$ & $\checkmark$ & \\
\hline Auto-ARIMA & richness & $\checkmark$ & $\checkmark$ & \\
\hline \multicolumn{5}{|c|}{ Environmental models } \\
\hline GBM richness & richness & & & $\checkmark$ \\
\hline Stacked SDMs & species-level presence & & & $\checkmark$ \\
\hline Mistnet JSDM & species composition & & & $\checkmark$ \\
\hline
\end{tabular}

100

our uncertainty in the true values of those effects. All of these models use a Gaussian error distribution (rather than a count distribution) for reasons discussed below (see "Model evaluation").

Baseline models. We used two simple baseline models as a basis for comparison with the more complex models (Figure 2A). The first baseline, called the "average" model, treated site-level richness observations as uncorrelated noise around a site-level constant:

$$
y_{t}=\mu+\epsilon_{t} .
$$

Predictions from the "average" model are thus centered on $\mu$, which could either be the mean of the raw training richness values, or an output from the observer model. This model's confidence intervals have a constant width that depends on the standard 
deviation of $\epsilon$, which can either be the standard deviation of the raw training richness

values, or $\sigma^{\text {residual }}$ from the observer model; see supplement).

The second baseline, called the "naive" model (Hyndman and Athanasopoulos 2014), was a simple autoregressive process with a single year of history, i.e. an $\operatorname{ARIMA}(0,1,0)$ model:

$$
y_{t}=y_{t-1}+\epsilon_{t}
$$

where the standard deviation of $\epsilon$ is a free parameter for each site. In contrast to the "average" model, whose predictions are based on the average richness across the whole time series, the "naive" model predicts that future observations will be similar to the final observed value (e.g., in our hindcasts the value observed in 2003). Moreover, because the $\epsilon$ values accumulate over time, the confidence intervals expand rapidly as the predictions extend farther into the future. Despite these differences, both models' richness predictions are centered on a constant value, so neither model can anticipate any trends in richness or any responses to future environmental changes.

Time series models. We used Auto-ARIMA models (based on the auto. arima function in the package forecast; Hyndman 2017) to represent an array of different time-series modeling approaches. These models can include an autoregressive component (as in the "naive" model, but with the possibility of longer-term dependencies in the underlying process), a moving average component (where the noise ean have serial autecorrelation) and an integration/differencing component (so that the analysis could be performed on sequential differences of the raw data, accommodating more complex patterns including trends). The auto. arima function chooses whether to include each of these components (and how many terms to include for each one) using AICc (Hyndman 2017). Since there is no seasonal component to the BBS time-series, we did not include a season component in these models. Otherwise we used the default settings for this function (See supplement for details). 


\section{Models: environmental models}

In contrast to the single-site models, most attempts to predict species richness focus on using correlative models based on environmental variables. We tested three common variants of this approach: direct modeling of species richness; stacking individual species distribution models; and joint species distribution models (JSDMs). Following the standard approach, site-level random effects were not included in these models as predictors, meaning that this approach implicitly assumes that two sites with identical Bioclim, elevation, and NDVI values should have identical richness distributions. As above, we included observer effects and the associated uncertainty by running these models 500 times (once per MCMC sample).

“Macroecological” model: richness GBM. We used a boosted regression tree model using the gbm package (Ridgeway et al. 2017) to directly model species richness as a function of environmental variables. Boosted regression trees are a form of tree-based modeling that work by fitting thousands of small tree-structured models sequentially, with each tree optimized to reduce the error of its predecessors. They are flexible models that are considered well suited for prediction (Elith et al. 2008). This model was optimized using a Gaussian likelihood, with a maximum interaction depth of 5, shrinkage of 0.015 , and up to 10,000 trees. The number of trees used for prediction was selected using the "out of bag" estimator; this number averaged 6,700 for the non-observer data and 7,800 for the observer-corrected data.

Species Distribution Model: stacked random forests. Species distribution models (SDMs) predict individual species' occurrence probabilities using environmental variables. Species-level models are used to predict richness by summing the predicted probability of occupancy across all species at a site. This avoids known problems with the use of thresholds for determining whether or not a species will be present at a site (Pellissier et al. 2013, Calabrese et al. 2014). Following Calabrese et al. (2014), we calculated the uncertainty in our richness estimate by treating richness as a sum over 
independent Bernoulli random variables: $\sigma_{\text {richness }}^{2}=\sum_{i} p_{i}\left(1-p_{i}\right)$, where $i$ indexes species. By itself, this approach is known to underestimate the true community-level uncertainty because it ignores the uncertainty in the species-level probabilites (Calabrese et al. 2014). To mitigate this problem, we used an ensemble of 500 estimates for each of the species-level probabilities instead of just one, propagating the uncertainty forward. We obtained these estimates using random forests (Liaw and Wiener 2002), a common approach in the species distribution modeling literature. Random forests are constructed by fitting hundreds of independent regression trees to randomly-perturbed versions of the data (Cutler et al. 2007, Caruana et al. 2008). When correcting for observer effects, each of the 500 trees in our species-level random forests used a different Monte Carlo estimate of the observer effects as a predictor variable.

Joint Species Distribution Model: mistnet. Joint species distribution models (JSDMs) are a new approach that makes predictions about the full composition of a community instead of modeling each species independently as above (Warton et al. 2015). JSDMs remove the assumed independence among species and explicitly account for the possibility that a site will be much more (or less) suitable for birds in general (or particular groups of birds) than one would expect based on the available environmental measurements alone. As a result, JSDMs do a better job of representing uncertainty about richness than stacked SDMs (Harris 2015, Warton et al. 2015). We used the mistnet package (Harris 2015) because it is the only JSDM that describes species' environmental associations with nonlinear functions.

\section{Model evaluation}

We defined model performance for all models in terms of continuous Gaussian errors, instead of using discrete count distributions. Variance in species richness within sites was lower than predicted by several common count models, such as the Poisson or binomial (i.e. richness was underdispersed for individual sites), so these count models 
oos would have had difficulty fitting the data (cf. Calabrese et al. 2014). The use of a

continuous distribution is adequate here, since richness had a relatively large mean (51) and all models produce continuous richness estimates. When a model was run multiple times for the purpose of correcting for observer effects, we used the mean of those runs' point estimates as our final point estimate and we calculated the uncertainty using the law of total variance (i.e. $\operatorname{Var}(\bar{y})+\mathbb{E}[\operatorname{Var}(y)]$, or the variance in point estimates plus the average residual variance).

We evaluated each model's forecasts using the data for each year between 2004 and 2013. We used three metrics for evaluating performance: 1) root-mean-square error (RMSE) to determine how far, on average, the models' predictions were from the observed value; 2) the $95 \%$ prediction interval coverage to determine how well the models predicted the range of possible outcomes; and 3) deviance (i.e. negative 2 times the Gaussian log-likelihood) as an integrative measure of fit that incorporates both accuracy and uncertainty. In addition to evaluating forecast performance in general, we evaluated how performance changed as the time horizon of forecasting increased by plotting performance metrics against year. Finally, we decomposed each model's squared error into two components: the squared error associated with site-level means and the squared error associated with annual fluctuations in richness within a site. This decomposition describes the extent to which each model's error depends on consistent differences among sites versus changes in site-level richness from year to year.

All analyses were conducted using R (R Core Team 2017). Primary R packages used in the analysis included dplyr (Wickham et al. 2017), tidyr (Wickham 2017), gimms (Detsch 2016), sp (Pebesma and Bivand 2005, Bivand et al. 2013), raster (Hijmans 2016), prism (PRISM Climate Group 2004), rdataretriever (McGlinn et al. 2017), forecast (Hyndman and Khandakar 2008, Hyndman 2017), git2r (Widgren and others 2016), ggplot (Wickham 2009), mistnet (Harris 2015), viridis (Garnier 2017), rstan (Stan Development Team 2016), yaml (Stephens 2016), purrr (Henry and Wickham 
2017), gbm (Ridgeway et al. 2017), randomForest (Liaw and Wiener 2002). Code to

fully reproduce this analysis is available on GitHub

(https://github.com/weecology/bbs-forecasting) and archived on Zenodo (Harris et al. 2017a).

\section{Results}

The site-observer mixed model found that $70 \%$ of the variance in richness in the training set could be explained by differences among sites, and $21 \%$ could be explained by differences among observers. The remaining $9 \%$ represents residual variation, where a given observer might report a different number of species in different years. In the training set, the residuals had a standard deviation of about 3.6 species. After correcting for observer differences, there was little temporal autocorrelation in these residuals (i.e. the residuals in one year explain $1.3 \%$ of the variance in the residuals of the following year), suggesting that richness was approximately stationary between 1982 and 2003.

When comparing forecasts for richness across sites all methods performed well (Figure 3; all $R^{2}>0.5$ ). However SDMs (both stacked and joint) and the macroecological model all failed to successfully forecast the highest-richness sites, resulting in a notable clustering of predicted values near $\sim 60$ species and the poorest model performance ( $R^{2}=0.52-0.78$, versus $R^{2}=0.67-0.87$ for the within-site methods).

While all models generally performed well in absolute terms (Figure 3), none consistently outperformed the "average" baseline (Figure 4). The auto-ARIMA was generally the best-performing non-baseline model, but in many cases (67\% of the time),

the auto.arima procedure selected a model with only an intercept term (i.e. no autoregressive terms, no drift, and no moving average terms), making it similar to the "average" model. All five alternatives to the "average" model achieved lower error on some of the sites in some years, but each one had a higher mean absolute error and 


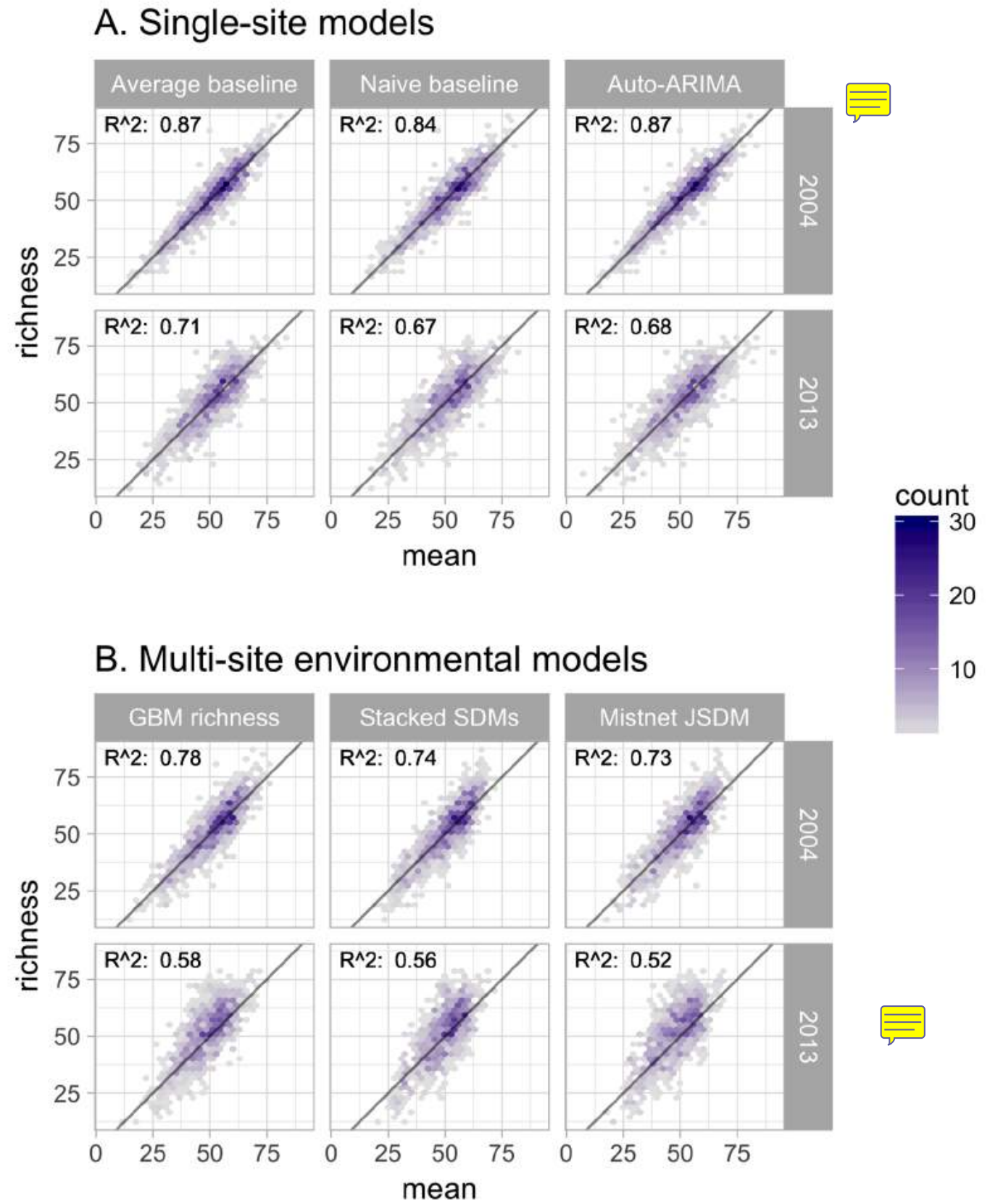

Figure 3: Performance of six forecasting models for predicting species richness one year (2004) and ten years into the future (2013). Plots show observed vs. predicted values for species richness. Models were trained with data from 1982-2003. In general, the single-site models (A) outperformed the environmental models $(\mathbf{B})$. The accuracy of the predictions generally declined as the timescale of the forecast was extended from 2004 to 2013. 
higher mean deviance (Figure 4).

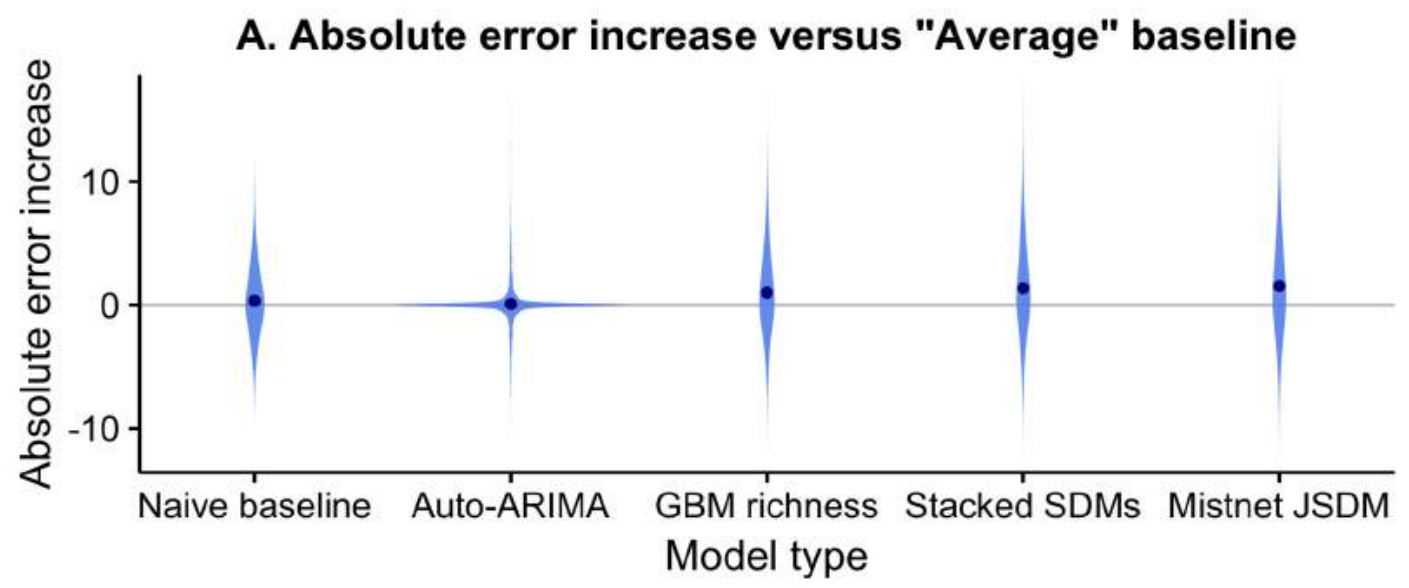

B. Deviance increase versus "Average" baseline

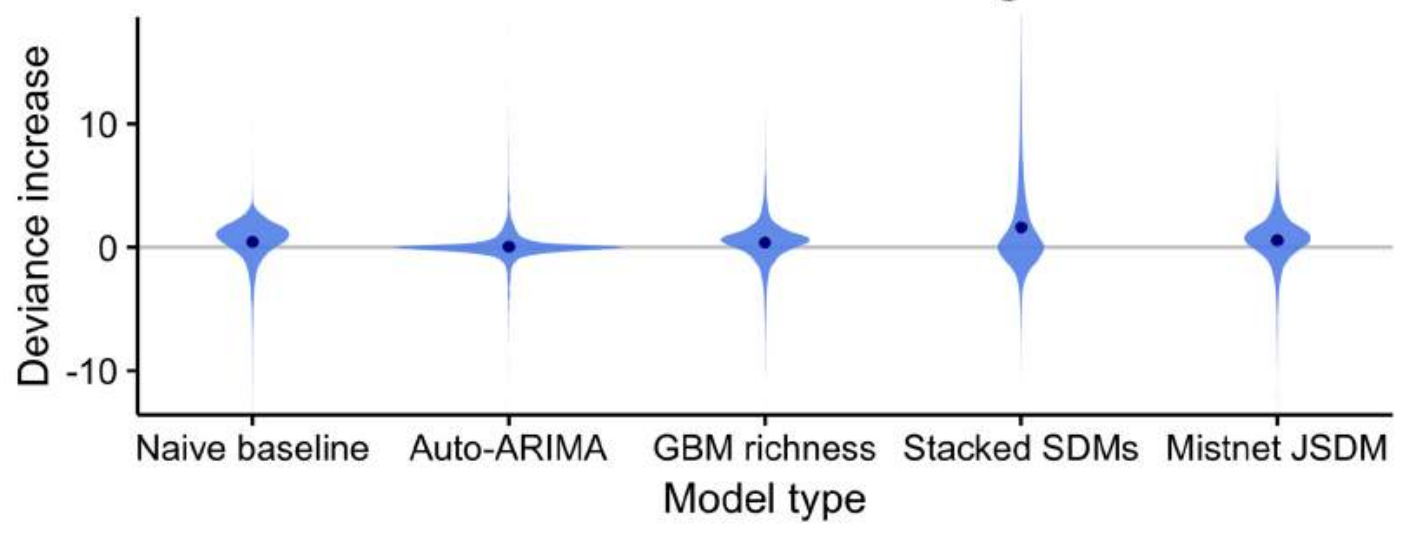

Figure 4: Difference between the forecast error of models and the error of the average baseline using both absolute error (A.) and deviance (B.). Differences are taken for each site and testing year so that errors for the same forecast are directly compared. The error of the average baseline is by definition zero and is indicated by the horizontal gray line. None of the five models provided a consistent improvement over the average baseline. The absolute error of the models was generally similar or larger than that of the "average" model, with large outliers in both directions. The deviance of the models was also generally higher than the "average" baseline.

Most models produced confidence intervals that were too narrow, indicating overconfident predictions (Figure 5C). The random forest-based SDM stack was the most overconfident model, with only $72 \%$ of observations falling inside its $95 \%$ confidence intervals. This stacked SDM's narrow predictive distribution caused it to have notably higher deviance (Figure 5B) than the next-worst model, even though its point estimates were not unusually bad in terms of RMSE (5A). As discussed elsewhere 


\section{A. Root mean squared error (RMSE)}

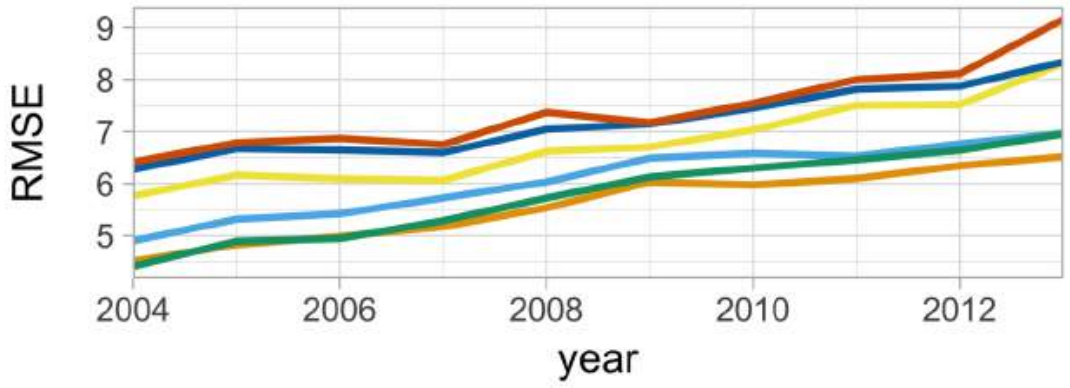

B. Coverage

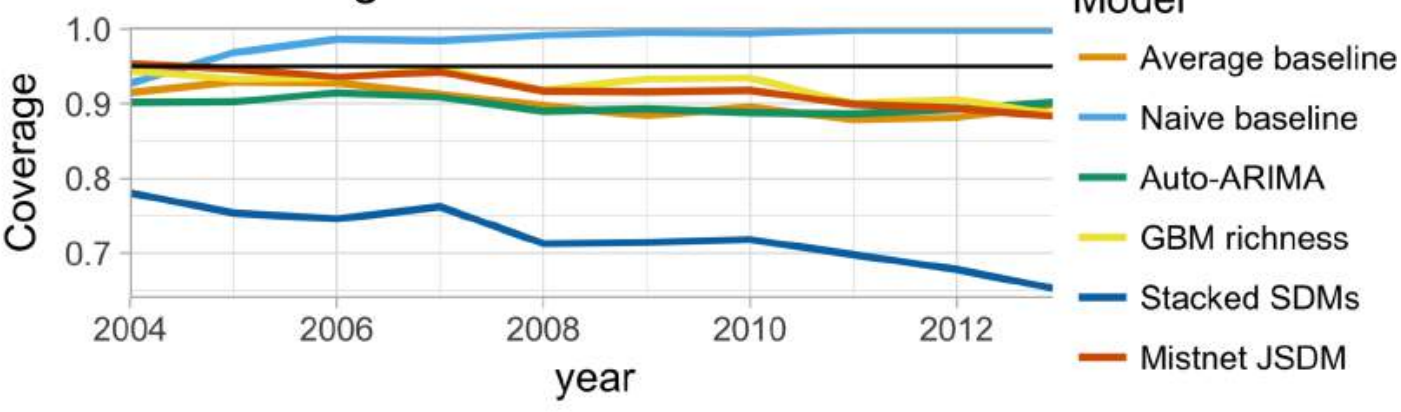

C. Mean deviance

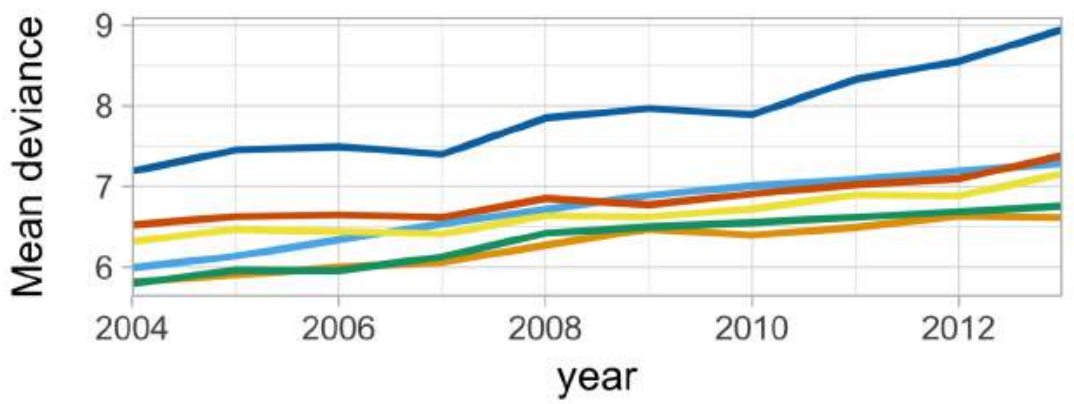

Figure 5: Change in performance of the six forecasting models with the time scale of the forecast (1-10 years into the future). A. Root mean square error (rmse; the error in the point estimates) shows the three environmental models tending to show the largest errors at all time scales and the models getting worse as they forecast further into the future at approximately the same rate. B. Deviance (lack of fit of the entire predictive distribution) shows the stacked species distribution models with much higher error than other models and shows that the "naive" model's deviance grows relatively quickly. C. Coverage of a model's 95\% confidence intervals (how often the observed values fall inside the predicted range; the black line indicates ideal performance) shows that the "naive" model's predictive distribution is too wide (capturing almost all of the data) and the stacked SDM's predictive distribution is too narrow (missing almost a third of the observed richness values by 2014). 
(Harris 2015), this overconfidence is a product of the assumption in stacked SDMs that errors in the species-level predictions are independent. The GBM-based "macroecological" model and the mistnet JSDM had the best calibrated uncertainty estimates (Figure 5B)and therefore their relative performance was higher in terms of deviance than in terms of RMSE. The "naive" model was the only model whose confidence intervals were too wide (Figure 5C), which can be attributed to the rapid rate at which these intervals expand (Figure 1).

Partitioning each model's squared error shows that the majority of the residual error was attributed to errors in estimating site-level means, rather than errors in tracking year-to-year fluctuations (Figure 6). The "average" model, which was based entirely on site-level means, had the lowest error in this regard. In contrast, the three environmental models showed larger biases at the site level, though they still explained most of the variance in this component. This makes sense, given that they could not explicitly distinguish among sites with similar climate, NDVI, and elevation. Interestingly, the environmental models had higher squared error than the baselines did for tracking year-to-year fluctuations in richness as well.

Accounting for differences among observers generally improved measures of model fit (Figure 7). Improvements primarily resulted from a small number of forecasts where observer turnover caused a large shift in the reported richness values. The naive baseline was less sensitive to these shifts, because it largely ignored the richness values reported by observers that had retired by the end of the training period (Figure 1). The average model, which gave equal weight to observations from the whole training period, showed a larger decline in performance when not accounting for observer effects especially in terms of coverage. The performance of the mistnet JSDM was notable here, because its prediction intervals retained good coverage even when not correcting for observer differences, which we attribute to the JSDM's ability to model this variation with its latent variables. 


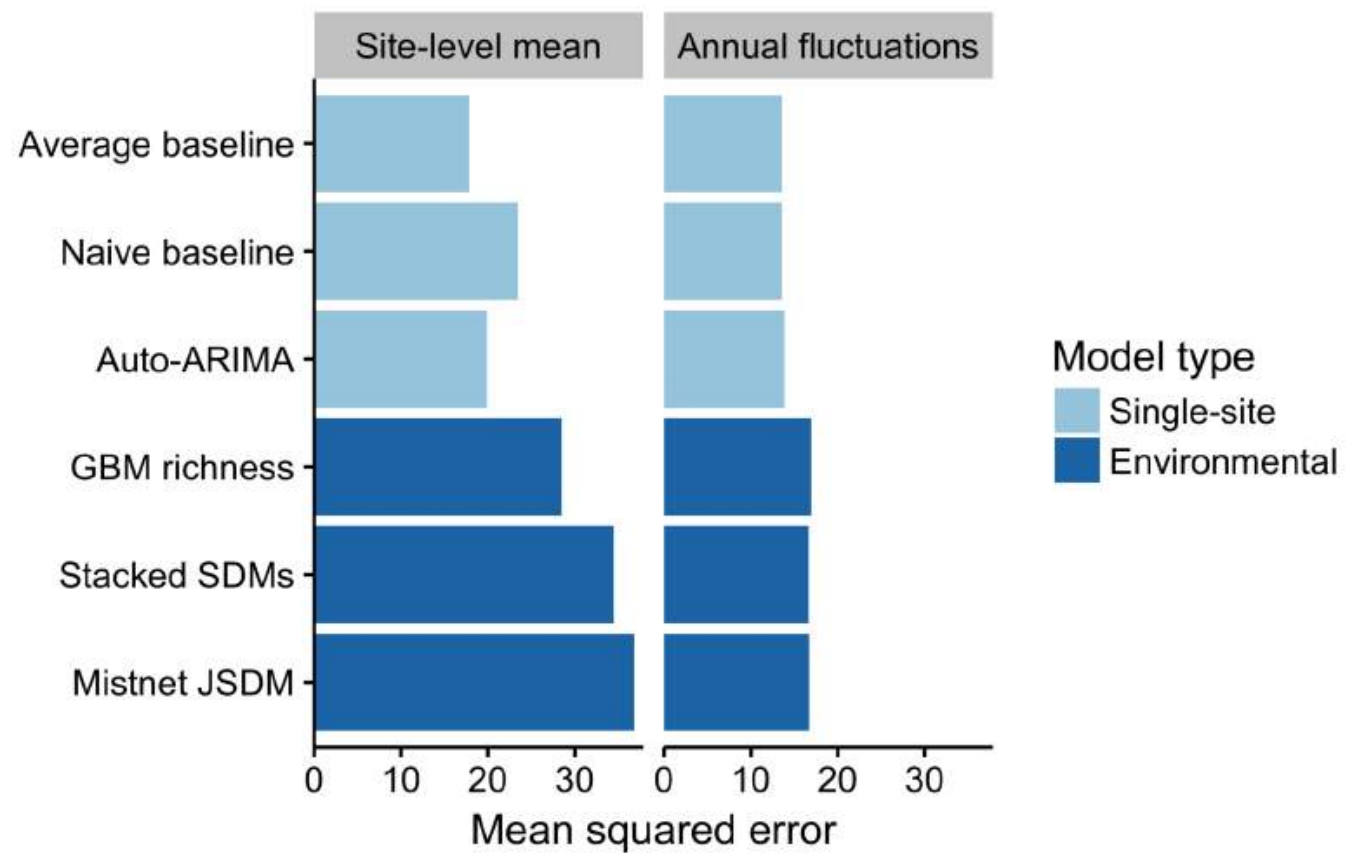

Figure 6: Partitioning of the squared error for each model into site and year components. The site-level mean component shows consistent over or under estimates of richness at a site across years. The annual fluctuation component shows errors in predicting fluctuations in a site's richness over time. Both components of the mean squared error were lower for the single-site models than for the environmental models. 


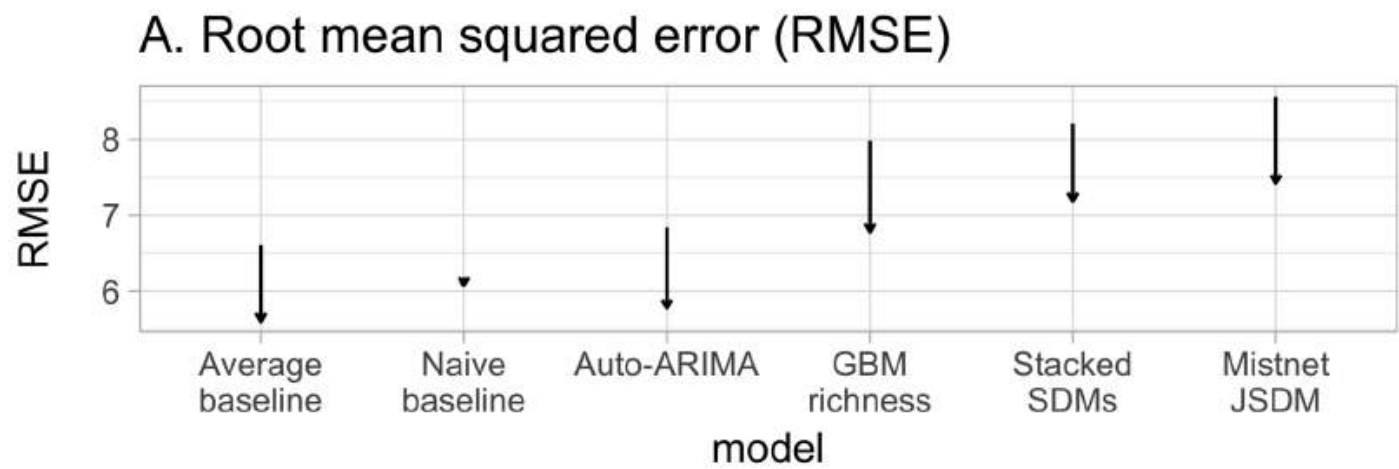

B. Coverage

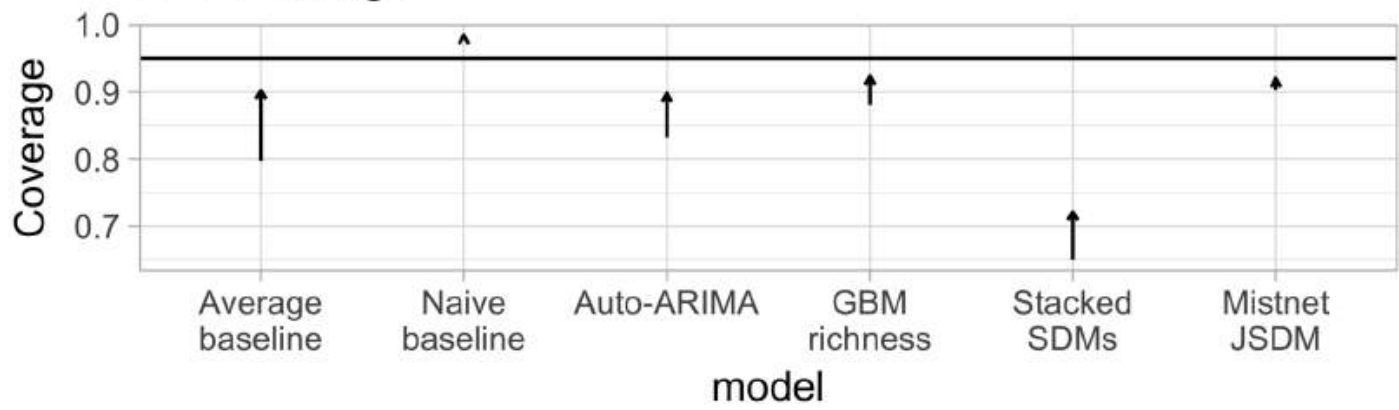

\section{Mean deviance}

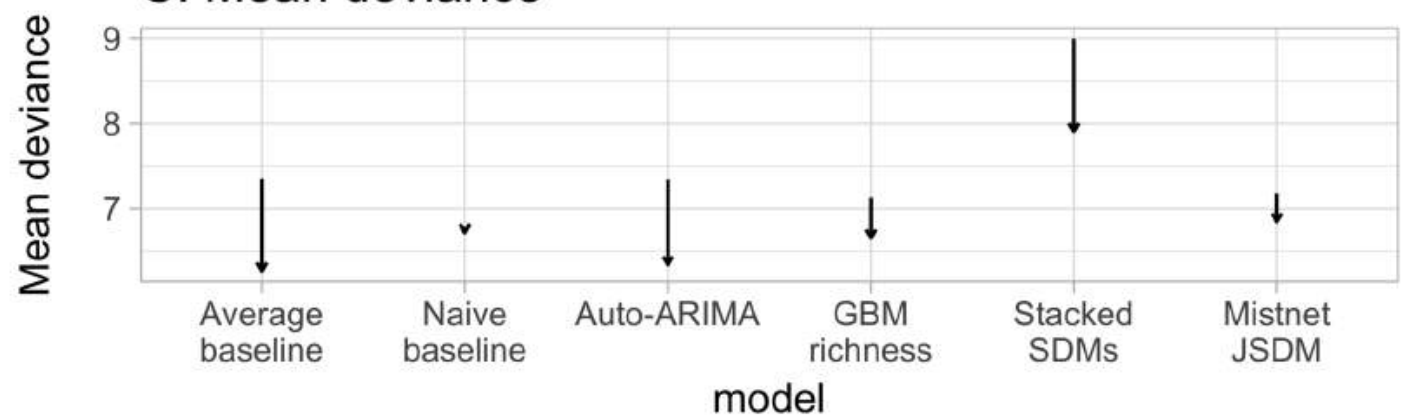

Figure 7: Controlling for differences among observers generally improved each model's predictions, on average. The magnitude of this effect was negligible for the Naive baseline, however. 


\section{Discussion}

Forecasting is an emerging imperative in ecology; as such, the field needs to develop and follow best practices for conducting and evaluating ecological forecasts (Clark et al. 2001). We have used a number of these practices (Box 1) in a single study that builds and evaluates forecasts of biodiversity in the form of species richness. The results of this effort are both promising and humbling. When comparing predictions across sites, many different approaches produce reasonable forecasts (Figure 3). If a site is predicted to have a high number of species in the future, relative to other sites, it generally does. However, none of the methods evaluated reliably determined how site-level richness changes over time (Figure 6), which is generally the stated purpose of these forecasts. As a result, baseline models, which did not attempt to anticipate changes in richness over time, generally provided the best forecasts for future biodiversity. While this study is restricted to breeding birds in North America, its results are consistent with a growing literature on the limits of ecological forecasting, as discussed below.

The most commonly used methods for forecasting future biodiversity, SDMs and macroecological models, both produced worse forecasts than time-series models and simple baselines. This weakness suggests that predictions about future biodiversity change should be viewed with skepticism unless the underlying models have been validated temporally, via hindcasting and comparison with simple baselines. Since site-level richness is relatively stable, spatial validation is not enough: a model can have high accuracy across spatiol gradients without being able to predict changes over time, This gap between spatial and temporal accuracy is known to be important for species-level predictions (Rapacciuolo et al. 2012, Oedekoven et al. 2017); our results indicate that it is substantial for higher-level patterns like richness as well. SDMs' poor temporal predictions are particularly sobering, as these models have been one of the main foundations for estimates of the predicted loss of biodiversity to climate change over the past decade or so $\equiv$ omas et al. 2004, Thuiller et al. 2011, Urban 2015). Our 
results also highlight the importance of comparing multiple modeling approaches when conducting ecological forecasts, and in particular, the value of comparing results to simple baselines to avoid over-interpreting the information present in these forecasts [Box 1]. Disciplines that have more mature forecasting cultures often do this by reporting "forecast skill", i.e., the improvement in the forecast relative to a simple baseline (Jolliffe and Stephenson 2003). We recommend following the example of Perretti et al. (2013) and adopting this approach in future ecological forecasting research.

When comparing different methods for forecasting our results demonstrate the importance of considering uncertainty (Box 1; Clark et al. 2001, Dietze et al. 2016). Previous comparisons between stacked SDMs and macroecological models reported that the methods yielded equivalent results for forecasting diversity (Algar et al. 2009, Distler et al. 2015). While our results support this equivalence for point estimates, they also show that stacked SDMs dramatically underestimate the range of possible outcomes; after ten years, more than a third of the observed richness values fell outside the stacked SDMs' 95\% prediction intervals. Consistent with Harris (2015) and Warton et al. (2015), we found that JSDMs' wider prediction intervals enabled them to avoid this problem. Macroecological models appear to share this advantage, while being considerably easier to implement.

We have only evaluated annual forecasts up to a decade into the future, but forecasts are often made with a lead time of 50 years or more. These long-term forecasts are difficult to evaluate given the small number of century-scale datasets, but are important for understanding changes in biodiversity at some of the lead times relevant for conservation and management. Two studies have assessed models of species richness at longer lead times (Algar et al. 2009, Distler et al. 2015), but the results were not compared to baseline or time-series models (in part due to data limitations) making them difficult to compare to our results directly. Studies on shorter time scales, such as 
ours, provide one way to evaluate our forecasting methods without having to wait several decades to observe the effects of environmental change on biodiversity (Petchey et al. 2015, Dietze et al. 2016, Tredennick et al. 2016), but cannot fully replace longer-term evaluations (Tredennick et al. 2016). In general, drivers of species richness can differ at different temporal scales (Rosenzweig 1995, White 2004, 2007, Blonder et al. 2017), so different methods may perform better for different lead times. In particular, we might expect environmental and ecological information to become more important at longer time scales, and thus for the performance of simple baseline forecasts to degrade faster than forecasts from SDMs and other similar models. We did observe a small trend in this direction: deviance for the auto-ARIMA models and for the average baseline grew faster than for two of the environmental models (the JSDM and the macroecological model), although this growth was not statistically significant for the average baseline.

While it is possible that models that include species' relationships to their environments or direct environmental constraints on richness will provide better fits at longer lead times, it is also possible that they will continue to produce forecasts that are worse than baselines that assume the systems are static. This would be expected to occur if richness in these systems is not changing over the relevant multi-decadal time scales, which would make simpler models with no directional change more appropriate. Recent suggestions that local scale richness in some systems is not changing directionally at multi-decadal scales supports this possibility (Brown et al. 2001, Ernest and Brown 2001, Vellend et al. 2013, Dornelas et al. 2014). A lack of change in richness may be expected even in the presence of substantial changes in environmental conditions and species composition at a site due to replacement of species from the regional pool (Brown et al. 2001, Ernest and Brown 2001). On average, the Breeding Bird Survey sites used in this study show little change in richness (site-level SD of 3.6 species, after controlling for differences among observers; see also La Sorte and Boecklen 2005). The absence of rapid change in this dataset is beneficial for the absolute accuracy of 
forecasts across different sites: when a past year's richness is already known, it is easy to estimate future richness. Ward et al. (2014) found similar patterns in time series of fisheries stocks, where relatively stable time series were best predicted by simple models and more complex models were only beneficial with dynamic time series. The site-level stability of the BBS data also explains why SDMs and macroecological models perform relatively well at predicting future richness, despite failing to capture changes in richness over time.

The relatively stable nature of the BBS richness time-series also makes it difficult to improve forecasts relative to simple baselines, since those baselines are already close to representing what is actually occurring in the system. It is possible that in systems exhibiting directional changes in richness and other biodiversity measures that models based on spatial patterns may yield better forecasts. Future research in this area should determine if regions or time periods exhibiting strong directional changes in biodiveristy are better predicted by these models and also extend our forecast horizon analyses to longer timescales where possible. Our results also suggest that future efforts to understand and forecast biodiversity should incorporate species composition, since lower-level processes are expected to be more dynamic (Ernest and Brown 2001, Dornelas et al. 2014) and contain more information about how the systems are changing (Harris 2015). More generally, determining the forecastability of different aspects of ecological systems under different conditions is an important next step for the future of ecological forecasting.

Future biodiversity forecasting efforts also need to address the uncertainty introduced by the error in forecasting the environmental conditions that are used as predictor variables. In this, and other hindcasting studies, the environmental conditions for the "future" are known because the data has already been observed. However, in real forecasts the environmental conditions themselves have to be predicted, and environmental forecasts will also have uncertainty and bias. Ultimately, ecological 
forecasts that use environmental data will therefore be more uncertain than our current

hindcasting efforts, and it is important to correctly incorporate this uncertainty into our models (Clark et al. 2001, Dietze 2017). Limitations in forecasting future

environmental conditions—particularly at small scales—will present continued challenges for models incorporating environmental variables, and this may result in a continued advantage for simple single-site approaches.

In addition to comparing and improving the process models used for forecasting it is important to consider the observation models. When working with any ecological dataset, there are imperfections in the sampling process that have the potential to influence results. With large scale surveys and citizen science datasets, such as the Breeding Bird Survey, these issues are potentially magnified by the large number of different observers and by major differences in the habitats and species being surveyed (Sauer et al. 1994). Accounting for differences in observers reduced the average error in our point estimates and also improved the coverage of the confidence intervals. In addition, controlling for observer effects resulted in changes in which models performed best, most notably improving most models' point estimates relative to the naive baseline. This demonstrates that modeling observation error can be important for properly estimating and reducing uncertainty in forecasts and can also lead to changes in the best methods for forecasting [Box 1]. This suggests that, prior to accounting for observer effects, the naive model performed well largely because it was capable of accommodating rapid shifts in estimated richness introduced by changes in the observer. These kinds of rapid changes were difficult for the other single-site models to accommodate. Another key aspect of an ideal observation model is imperfect detection. In this study, we did not address differences in detection probability across species and sites (Boulinier et al. 1998) since there is no clear way to address this issue using North American Breeding Bird Survey data without making strong assumptions about the data (i.e., assuming there is no biological variation in stops along a route; White and Hurlbert 2010), but this would be a valuable addition to future forecasting models. 
The science of forecasting biodiversity remains in its infancy and it is important to consider weaknesses in current forecasting methods in that context. In the beginning, weather forecasts were also worse than simple baselines, but these forecasts have continually improved throughout the history of the field (McGill 2012, Silver 2012, Bauer et al. 2015). One practice that lead to improvements in weather forecasts was that large numbers of forecasts were made publicly, allowing different approaches to be regularly assessed and refined (McGill 2012, Silver 2012). To facilitate this kind of improvement, it is important for ecologists to start regularly making and evaluating real ecological forecasts, even if they perform poorly, and to make these forecasts openly available for assessment (McGill 2012, Dietze et al. 2016). These forecasts should include both short-term predictions, which can be assessed quickly, and mid- to long-term forecasts, which can help ecologists to assess long time-scale processes and determine how far into the future we can successfully forecast (Dietze et al. 2016, Tredennick et al. 2016). We have openly archived forecasts from all six models through the year 2050 (Harris et al. 2017b), so that we and others can assess how well they perform. We plan to evaluate these forecasts and report the results as each new year of BBS data becomes available, and make iterative improvements to the forecasting models in response to these assessments.

Making successful ecological forecasts will be challenging. Ecological systems are complex, our fundamental theory is less refined than for simpler physical and chemical systems, and we currently lack the scale of data that often produces effective forecasts through machine learning. Despite this, we believe that progress can be made if we develop an active forecasting culture in ecology that builds and assesses forecasts in ways that will allow us to improve the effectiveness of ecological forecasts more rapidly (Box 1; McGill 2012, Dietze et al. 2016). This includes expanding the scope of the ecological and environmental data we work with, paying attention to uncertainty in both model building and forecast evaluation, and rigorously assessing forecasts using a combination of hindcasting, archived forecasts, and comparisons to simple baselines. 
517

\section{Acknowledgments}

We thank the developers and providers of the data and software that made this research possible including: the PRISM Climate Group at Oregon State University, the staff at USGS and volunteer citizen scientists associated with the North American Breeding Bird Survey, NASA, the World Climate Research Programme's Working Group on Coupled Modelling and its working groups, the U.S. Department of Energy's Program for Climate Model Diagnosis and Intercomparison, and the Global Organization for Earth System Science Portals. A. C. Perry provided valuable comments that improved the clarity of this manuscript.

\section{Box 1: Best practices for making and evaluating ecological forecasts}

\section{Compare multiple modeling approaches}

Typically ecological forecasts use one modeling approach or a small number of related approaches. By fitting and evaluating multiple modeling approaches we can learn more

rapidly about the best approaches for making predictions for a given ecological quantity (Clark et al. 2001, Ward et al. 2014). This includes comparing process-based (e.g., Kearney and Porter 2009) and data-driven models (e.g., Ward et al. 2014), as well as comparing the accuracy of forecasts to simple baselines to determine if the modeled forecasts are more accurate than the naive assumption that the world is static (Jolliffe and Stephenson 2003, Perretti et al. 2013).

\section{Use time-series data when possible}

Forecasts describe how systems are expected to change through time. While some areas of ecological forecasting focus primarily on time-series data (Ward et al. 2014), others primarily focus on using spatial models and space-for-time substitutions (Blois et al. 
540

541

542

2013). Using ecological and environmental time-series data allows the consideration of actual dynamics from both a process and error structure perspective (Tredennick et al. 2016).

\section{Pay attention to uncertainty}

Understanding uncertainty in a forecast is just as important as understanding the average or expected outcome. Failing to account for uncertainty can result in overconfidence in uncertain outcomes leading to poor decision making and erosion of confidence in ecological forecasts (Clark et al. 2001). Models should explicitly include sources of uncertainty and propagate them through the forecast where possible (Clark et al. 2001, Dietze 2017). Evaluations of forecasts should assess the accuracy of models' estimated uncertainties as well as their point estimates (Dietze 2017).

\section{Use predictors related to the question}

Many ecological forecasts use data that is readily available and easy to work with.

While ease of use is a reasonable consideration it is also important to include predictor variables that are expected to relate to the ecological quantity being forecast. Time-series of predictors, instead of long-term averages, are also preferable to match the ecologial data (see \#2). Investing time in identifying and acquiring better predictor variables may have at least as many benefits as using more sophisticated modeling techniques (Kent et al. 2014).

\section{Address unknown or unmeasured predictors}

Ecological systems are complex and many biotic and abiotic aspects of the environment are not regularly measured. As a result, some sites may deviate in consistent ways from model predictions. Unknown or unmeasured predictors can be incorporated in models 
563

564

using site-level random effects (potentially spatially autocorrelated) or by using latent variables that can identify unmeasured gradients (Harris 2015).

\section{Assess how forecast accuracy changes with time-lag}

In general, the accuracy of forecasts decreases with the length of time into the future being forecast (Petchey et al. 2015). This decay in accuracy should be considered when evaluating forecasts. In addition to simple decreases in forecast accuracy the potential for different rates of decay to result in different relative model performance at different lead times should be considered.

\section{Include an observation model}

Ecological observations are influenced by both the underlying biological processes (e.g. resource limitation) and how the system is sampled. When possible, forecasts should model the factors influencing the observation of the data (Yu et al. 2010, Hutchinson et al. 2011, Schurr et al. 2012).

\section{Validate using hindcasting}

Evalutating a model's predictive performance across time is critical for understanding if it is useful for forecasting the future. Hindcasting uses a temporal out-of-sample validation approach to mimic how well a model would have performed had it been run in the past. For example, using occurance data from the early 20th century to model distributions which are validated with late 20th century occurances. Dense time series, such as yearly observations, are desirable to also evalulate the forecast horizon (see \#6), but this is not a strict requirement. 


\section{Publicly archive forecasts}

Forecast values and/or models should be archived so that they can be assessed after new data is generated (McGill 2012, Silver 2012, Dietze et al. 2016). Enough information should be provided in the archive to allow unambiguous assessment of each forecast's performance (Tetlock and Gardner 2016).

\section{Make both short-term and long-term predictions}

Even in cases where long-term predictions are the primary goal, short-term predictions should also be made to accommodate the time-scales of planning and management decisions and to allow the accuracy of the forecasts to be quickly evaluated (Dietze et al. 2016, Tredennick et al. 2016).

\section{References}

Algar, A. C., H. M. Kharouba, E. R. Young, and J. T. Kerr. 2009. Predicting the future of species diversity: Macroecological theory, climate change, and direct tests of alternative forecasting methods. Ecography 32:22-33.

Bauer, P., A. Thorpe, and G. Brunet. 2015. The quiet revolution of numerical weather prediction. Nature 525:47-55.

Bivand, R. S., E. Pebesma, and V. Gomez-Rubio. 2013. Applied spatial data analysis with R, second edition. Springer, NY.

Blois, J. L., J. W. Williams, M. C. Fitzpatrick, S. T. Jackson, and S. Ferrier. 2013. Space can substitute for time in predicting climate-change effects on biodiversity 110:9374-9379.

Blonder, B., D. E. Moulton, J. Blois, B. J. Enquist, B. J. Graae, M. Macias-Fauria, B. McGill, S. Nogué, A. Ordonez, B. Sandel, and J.-C. Svenning. 2017. Predictability in 
community dynamics. Ecology Letters 20:293-306.

Boulinier, T., J. D. Nichols, J. R. Sauer, J. E. Hines, and K. Pollock. 1998. Estimating species richness: The importance of heterogeneity in species detectability. Ecology 79:1018-1028.

Brekke, L., B. Thrasher, E. Maurer, and T. Pruitt. 2013. Downscaled cMIP3 and cMIP5 climate and hydrology projections: Release of downscaled cMIP5 climate projections, comparison with preceding information, and summary of user needs. US Dept. of the Interior, Bureau of Reclamation, Technical Services Center, Denver.

Brown, J. H., S. Ernest, J. M. Parody, and J. P. Haskell. 2001. Regulation of diversity: Maintenance of species richness in changing environments. Oecologia 126.

Calabrese, J. M., G. Certain, C. Kraan, and C. F. Dormann. 2014. Stacking species distribution models and adjusting bias by linking them to macroecological models. Global Ecology and Biogeography 23:99-112.

Cardinale, B. J., J. E. Duffy, A. Gonzalez, D. U. Hooper, C. Perrings, P. Venail, A. Narwani, G. M. Mace, D. Tilman, D. A. Wardle, and others. 2012. Biodiversity loss and its impact on humanity. Nature 486:59-67.

Carpenter, B., A. Gelman, M. D. Hoffman, D. Lee, B. Goodrich, M. Betancourt, M. Brubaker, J. Guo, P. Li, and A. Riddell. 2017. Stan : A Probabilistic Programming Language. Journal of Statistical Software 76.

Caruana, R., N. Karampatziakis, and A. Yessenalina. 2008. An empirical evaluation of supervised learning in high dimensions. Pages 96-103 in Proceedings of the 25th international conference on machine learning. ACM.

Clark, J. S., S. R. Carpenter, M. Barber, S. Collins, A. Dobson, J. A. Foley, D. M. Lodge, M. Pascual, R. Pielke, W. Pizer, and others. 2001. Ecological forecasts: An emerging imperative. Science 293:657-660.

Cutler, D. R., T. C. Edwards, K. H. Beard, A. Cutler, K. T. Hess, J. Gibson, and J. J. 
633

634

Lawler. 2007. Random forests for classification in ecology. Ecology 88:2783-2792.

Detsch, F. 2016. Gimms: Download and process gIMMS nDVI3g data. R package version 1.0.0.

Dietze, M. C. 2017. Ecological forecasting. Princeton University Press.

Dietze, M. C., A. Fox, J. L. Betancourt, M. B. Hooten, C. S. Jarnevich, T. H. Keitt, M.

Kenney, C. Laney, L. Larsen, H. W. Loescher, and others. 2016. Iterative ecological

forecasting: Needs, opportunities, and challenges. in NEON workshop:

Operationalizing ecological forecasting.

Distler, T., J. G. Schuetz, J. Velásquez-Tibatá, and G. M. Langham. 2015. Stacked species distribution models and macroecological models provide congruent projections of avian species richness under climate change. Journal of Biogeography 42:976-988.

Díaz, S., S. Demissew, J. Carabias, C. Joly, M. Lonsdale, N. Ash, A. Larigauderie, J. R. Adhikari, S. Arico, A. Báldi, and others. 2015. The iPBES conceptual framework-connecting nature and people. Current Opinion in Environmental Sustainability 14:1-16.

Dornelas, M., N. J. Gotelli, B. McGill, H. Shimadzu, F. Moyes, C. Sievers, and A. E. Magurran. 2014. Assemblage time series reveal biodiversity change but not systematic loss. Science 344:296-299.

Elith, J., J. R. Leathwick, and T. Hastie. 2008. A working guide to boosted regression trees. Journal of Animal Ecology 77:802-813.

Ernest, S. M., and J. H. Brown. 2001. Homeostasis and compensation: The role of species and resources in ecosystem stability. Ecology 82:2118-2132.

Garnier, S. 2017. viridis: Default color maps from 'matplotlib'. R package version 0.4.0.

Harris, D. J. 2015. Generating realistic assemblages with a joint species distribution model. Methods in Ecology and Evolution 6:465-473. 
Harris, D. J., E. White, and S. D. Taylor. 2017a. Weecology/bbs-forecasting. Zenodo.

DOI:10.5281/zenodo.888989.

Harris, D. J., E. White, and S. D. Taylor. 2017b. Weecology/forecasts: V0.0.2. Zenodo. DOI:10.5281/zenodo.1101123.

Henry, L., and H. Wickham. 2017. purrr: Functional programming tools. R package version 0.2.2.2.

Hijmans, R. J. 2016. raster: Geographic data analysis and modeling. R package version 2.5-8

Houlahan, J. E., S. T. McKinney, T. M. Anderson, and B. J. McGill. 2017. The priority of prediction in ecological understanding. Oikos 126:1-7.

Hurlbert, A. H., and J. P. Haskell. 2002. The effect of energy and seasonality on avian species richness and community composition. The American Naturalist 161:83-97.

Hurlbert, A. H., and E. P. White. 2005. Disparity between range map-and survey-based analyses of species richness: Patterns, processes and implications. Ecology Letters $8: 319-327$.

Hutchinson, R. A., L.-P. Liu, and T. G. Dietterich. 2011. Incorporating boosted regression trees into ecological latent variable models. Pages 1343-1348 in Proceedings of the twenty-fifth aAAI conference on artificial intelligence. San Francisco, California. Hyndman, R. J. 2017. forecast: Forecasting functions for time series and linear models. R package version 8.1.

Hyndman, R. J., and G. Athanasopoulos. 2014. Forecasting: Principles and practice. OTexts.

Hyndman, R. J., and Y. Khandakar. 2008. Automatic time series forecasting: The forecast package for R. Journal of Statistical Software 26:1-22.

IPCC. 2014. Summary for policymakers. in C. Field, V. Barros, D. Dokken, K. Mach, 
M. Mastrandrea, T. Bilir, M. Chatterjee, K. Ebi, Y. Estrada, R. Genova, B. Girma, E. Kissel, A. Levy, S. MacCracken, P. Mastrandrea, and L. White, editors. Climate change 2014: Impacts, adaptation, and vulnerability. Part A: Global and sectoral aspects.

Contribution of Working Group II to the Fifth Assessment Report of the Intergovernmental Panel on Climate Change. Cambridge University Press.

Jarvis, A., H. Reuter, A. Nelson, and E. Guevara. 2008. Hole-filled SRTM for the globe Version 4, available from the CGIAR-CSI SRTM 90m Database.

Jetz, W., D. S. Wilcove, and A. P. Dobson. 2007. Projected impacts of climate and land-use change on the global diversity of birds. PLoS biology 5:e157.

Jolliffe, I. T., and D. B. Stephenson, editors. 2003. Forecast verification: a practitioner's guide in atmospheric science. John Wiley; Sons, Ltd.

Kearney, M., and W. Porter. 2009. Mechanistic niche modelling: Combining physiological and spatial data to predict species' ranges. Ecology letters 12:334-350.

Kent, R., A. Bar-Massada, and Y. Carmel. 2014. Bird and mammal species composition in distinct geographic regions and their relationships with environmental factors across multiple spatial scales. Ecology and evolution 4:1963-1971.

La Sorte, F. A., and W. J. Boecklen. 2005. Changes in the diversity structure of avian assemblages in north america. Global Ecology and Biogeography 14:367-378.

Liaw, A., and M. Wiener. 2002. Classification and regression by randomForest. R News $2: 18-22$.

Maguire, K. C., D. Nieto-Lugilde, J. L. Blois, M. C. Fitzpatrick, J. W. Williams, S.

Ferrier, and D. J. Lorenz. 2016. Controlled comparison of species- and community-level models across novel climates and communities. Proceedings of the Royal Society B: Biological Sciences 283:20152817.

McGill, B. J. 2012. Ecologists need to do a better job of prediction - part iI - partly cloudy and a $20 \%$ chance of extinction (or the 6 p's of good prediction). 
McGlinn, D., H. Senyondo, S. Taylor, and E. White. 2017. rdataretriever: R interface to the data retriever. $\mathrm{R}$ package version 1.0.0.

Morris, B. D., and E. P. White. 2013. The ecoData retriever: Improving access to existing ecological data. PLOS One 8:e65848.

Oedekoven, C. S., D. A. Elston, P. J. Harrison, M. J. Brewer, S. T. Buckland, A. Johnston, S. Foster, and J. W. Pearce-Higgins. 2017. Attributing changes in the distribution of species abundance to weather variables using the example of british breeding birds. Methods in Ecology and Evolution.

Pardieck, K. L., D. J. Ziolkowski Jr, Lutmerding M, K. Campbell, and M.-A. Hudson. 2017. North american breeding bird survey dataset 1966 - 2016, version 2016.0. U.S.

Geological Survey, Patuxent Wildlife Research Center.

Pebesma, E. J., and R. S. Bivand. 2005. Classes and methods for spatial data in R. R News 5:9-13.

Pellissier, L., A. Espíndola, J.-N. Pradervand, A. Dubuis, J. Pottier, S. Ferrier, and A. Guisan. 2013. A probabilistic approach to niche-based community models for spatial forecasts of assemblage properties and their uncertainties. Journal of Biogeography 40:1939-1946.

Perretti, C. T., S. B. Munch, and G. Sugihara. 2013. Model-free forecasting outperforms the correct mechanistic model for simulated and experimental data.

Proceedings of the National Academy of Sciences 110:5253-5257.

Petchey, O. L., M. Pontarp, T. M. Massie, S. K. Efi, A. Ozgul, M. Weilenmann, G. M. Palamara, F. Altermatt, B. Matthews, J. M. Levine, D. Z. Childs, B. J. Mcgill, M. E. Schaepman, B. Schmid, P. Spaak, A. P. Beckerman, F. Pennekamp, and I. S. Pearse. 2015. The ecological forecast horizon, and examples of its uses and determinants. Ecology Letters 18:597-611.

Pinzon, J. E., and C. J. Tucker. 2014. A non-stationary 1981-2012 aVHRR nDVI3g 
time series. Remote Sensing 6:6929-6960.

PRISM Climate Group, O. S. U. 2004. PRISM gridded climate data.

http://prism.oregonstate.edu/.

R Core Team. 2017. R: A language and environment for statistical computing. R Foundation for Statistical Computing, Vienna, Austria.

Rapacciuolo, G., D. B. Roy, S. Gillings, R. Fox, K. Walker, and A. Purvis. 2012.

Climatic associations of british species distributions show good transferability in time but low predictive accuracy for range change. PLoS One 7:e40212.

Ridgeway, G., with contributions from others. 2017. gbm: Generalized boosted regression models. $\mathrm{R}$ package version 2.1.3.

Rosenzweig, M. L. 1995. Species diversity in space and time. Cambridge University Press.

Sauer, J. R., B. G. Peterjohn, and W. A. Link. 1994. Observer differences in the north american breeding bird survey. The Auk:50-62.

Schurr, F. M., J. Pagel, J. S. Cabral, J. Groeneveld, O. Bykova, R. B. O’Hara, F. Hartig, W. D. Kissling, H. P. Linder, G. F. Midgley, and others. 2012. How to understand species' niches and range dynamics: A demographic research agenda for biogeography. Journal of Biogeography 39:2146-2162.

Senyondo, H., B. D. Morris, A. Goel, A. Zhang, A. Narasimha, S. Negi, D. J. Harris, D. Gertrude Digges, K. Kumar, A. Jain, K. Pal, K. Amipara, and E. P. White. 2017.

Retriever: Data retrieval tool. The Journal of Open Source Software 2:451.

Silver, N. 2012. The signal and the noise: Why so many predictions fail-but some don't. Penguin.

Stan Development Team. 2016. RStan: The R interface to Stan. R package version 2.14.1. 
Stephens, J. 2016. yaml: Methods to convert $r$ data to yAML and back. R package version 2.1.14.

Tetlock, P. E., and D. Gardner. 2016. Superforecasting: The art and science of prediction. Random House.

Thomas, C. D., A. Cameron, R. E. Green, M. Bakkenes, L. J. Beaumont, Y. C.

Collingham, B. F. Erasmus, M. F. De Siqueira, A. Grainger, L. Hannah, and others. 2004. Extinction risk from climate change. Nature 427:145-148.

Thuiller, W., S. Lavergne, C. Roquet, I. Boulangeat, B. Lafourcade, and M. B. Araujo. 2011. Consequences of climate change on the tree of life in europe. Nature 470:531.

Tilman, D., M. Clark, D. R. Williams, K. Kimmel, S. Polasky, and C. Packer. 2017. Future threats to biodiversity and pathways to their prevention. Nature 546:73-81. Tredennick, A. T., M. B. Hooten, C. L. Aldridge, C. G. Homer, A. R. Kleinhesselink, and P. B. Adler. 2016. Forecasting climate change impacts on plant populations over large spatial extents. Ecosphere 7.

Urban, M. C. 2015. Accelerating extinction risk from climate change. Science 348:571-573.

Vellend, M., L. Baeten, I. H. Myers-Smith, S. C. Elmendorf, R. Beauséjour, C. D. Brown, P. De Frenne, K. Verheyen, and S. Wipf. 2013. Global meta-analysis reveals no net change in local-scale plant biodiversity over time. Proceedings of the National Academy of Sciences 110:19456-19459.

Ward, E. J., E. E. Holmes, J. T. Thorson, and B. Collen. 2014. Complexity is costly: A meta-analysis of parametric and non-parametric methods for short-term population forecasting. Oikos 123:652-661.

Warton, D. I., F. G. Blanchet, R. B. O'Hara, O. Ovaskainen, S. Taskinen, S. C. Walker, and F. K. Hui. 2015. So many variables: Joint modeling in community ecology. Trends in Ecology \& Evolution 30:766-779. 
787

788

789

White, E. P. 2004. Two-phase species-time relationships in north american land birds. Ecology Letters 7:329-336.

White, E. P. 2007. Spatiotemporal scaling of species richness: Patterns, processes, and implications. Scaling biodiversity (eds D. Storch, PA Marquet \& JH Brown):325-346.

White, E. P., and A. H. Hurlbert. 2010. The combined influence of the local

environment and regional enrichment on bird species richness. The American Naturalist 175:E35-E43.

Wickham, H. 2009. Ggplot2: Elegant graphics for data analysis. Springer-Verlag New York.

Wickham, H. 2017. Tidyr: Easily tidy data with 'spread()' and 'gather()' functions. R package version 0.6.3.

Wickham, H., R. Francois, L. Henry, and K. Müller. 2017. Dplyr: A grammar of data manipulation. $\mathrm{R}$ package version 0.7.1.

Widgren, S., and others. 2016. git2r: Provides access to git repositories. R package version 0.14.0.

Yu, J., W.-K. Wong, and R. A. Hutchinson. 2010. Modeling experts and novices in citizen science data for species distribution modeling. Pages 1157-1162 in Data mining (iCDM), 2010 iEEE 10th international conference on. IEEE. 